\title{
The Role of HnRNA in the Control of Ribosomal Gene Transcription
}

\author{
Constantine E. Sekeris \\ National Hellenic Research Foundation, Biological Research Centre, \\ Vassileos Konstantinou 48, Athens, Greece \\ (Received 16 October 1984, and in revised form 8 December 1984) \\ The hypothesis is put forward whereby a processed fragment of HnRNA \\ acts as activator of ribosomal gene transcription by base-pair interaction \\ with the non-transcribed region of the ribosomal gene.
}

Regulation of transcription has received considerable attention during the last two decades and various models of control have been proposed, implicating as regulatory molecules either proteins, positively or negatively affecting transcription by direct interaction with the genetic material (Jacob \& Monod, 1961), or RNA, modulating RNA synthesis by base-pair interaction with the DNA (Davidson \& Britten, 1979; Georgiev, 1969). Both models have been mainly concerned with enzyme induction in lower and higher organisms by inducers, such as metabolites and hormones, and refer mainly to messenger RNA regulation. In contrast to mRNA transcription control, rDNA expression, particularly in the eucaryotic cell, is not yet well understood, but is now receiving due attention (Nomura, Gourse \& Baugham, 1984). Schmid \& Sekeris (1973a) had postulated that a cleavage product of HnRNA could act as a regulatory signal for the control of rDNA transcription. In this paper this hypothesis will be reiterated and expanded and some recent findings from the literature that conform with it will be presented.

Niessing et al. (1970) observed that the administration of a-amanitin, an inhibitor of DNA-dependent RNA polymerase II, to rats, results not only in inhibition of HnRNA synthesis but also blocks synthesis of ribosomal RNA. This observation could be explained by assuming that a-amanitin inhibits the formation of a mRNA coding for a protein involved in rRNA transcription or that HnRNA is directly involved in transcription activation. Using the inhibitor of protein synthesis cycloheximide, and comparing the time sequence of cycloheximide versus a-amanitin inhibition of rRNA synthesis, it was shown that a-amanitin inhibits ribosomal gene transcription much more rapidly than does cycloheximide (Schmid \& Sekeris, 1973a), 
although both inhibitors reach intracellular active concentrations within the same time interval. Thus, it was demonstrated that the a-amanitin evoked inhibition of rRNA synthesis is independent of potential translational effects. This led to the conclusion that HnRNA is directly involved in rRNA transcription (Schmid \& Sekeris, 1973a). Another relevant finding involved the effects of glucocorticoids on RNA synthesis. It is known that these steroid hormones induce both HnRNA and rRNA synthesis (Karlson, Doenecke \& Sekeris, 1975). Furthermore, glucocorticoids act sequentially on RNA synthesis, stimulating first extranucleolar and, somewhat later, rRNA synthesis (Schmid \& Sekeris, 1973b). As the glucocorticoid-receptorcomplex binds to DNA sites in the vicinity of, or within, the glucocorticoid inducible structural genes (Govindan, Spiess \& Majors, 1982; Scheidereit et al., 1983), whereas no binding sites on rDNA have been reported, it is strongly suggested that the stimulation of rRNA synthesis by glucocorticoids is a secondary effect of the hormone, following and perhaps dependent on HnRNA synthesis. The model of control of rDNA transcription we proposed (Karlson et al., 1975; Schmid \& Sekeris, 1973a) was based on the prevailing knowledge at that time concerning the fate of HnRNA and on ignorance as regards the complicated processing mechanisms, such as splicing. We proposed that a specific stretch of the HnRNA, remaining after processing of the molecule, interacts by base pairing with a certain sequence of rDNA, the interaction resulting in enhanced rDNA transcription. We now know that the ribosomal genes in higher organisms are organized as tandem repeats, localized at the nucleolar organizing regions of the chromosomes. Each repeat contains a transcribed coding and spacer region and a nontranscribed spacer (NTS).

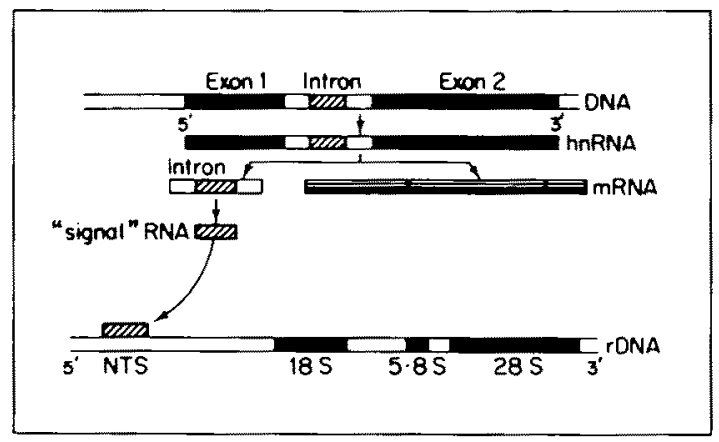

FIG. 1. The gene is transcribed into HnRNA, which is then processed into mRNA and "signal" RNA. The "signal" RNA then hybridizes to the NTS, either naked or in association with specific HnRNP proteins. In the present case the "signal" RNA is shown as part of the intron sequence, but could also be generated from other regions of HnRNA. 
Recent results from various laboratories point to the presence in the genome of several organisms of homologies to NTS sequences residing in regions outside the rDNA genes (Arnheim et al., 1980; Miesfeld, Krystal \& Arnheim, 1981; Mroiczka et al., 1984). Interestingly, sequences in a 6KB fragment of NTS from mouse, 5 - to the $45 \mathrm{~S}$ rRNA precursor, have been localized adjacent to the immunoglobulin heavy chain, flanking the $\mu, \alpha$ and $\gamma 2 b_{H}$ genes (Arnheim et al., 1980; Miesfeld, Krystal \& Arnheim, 1981). In addition, Miesfeld et al. (1981) localized human interspersed repeated sequences, hybridizing with NTS, in a 251 base pair segment between $\delta$ and $\beta$ globin genes. Similar sequences homologous to mouse NTS were found in the genomes of Xenopus, pigeon, slime mold and yeast.

In a recent paper, Mroiczka et al. (1984) detected sequences of the NTS of rat, both $5^{\prime}$ to the external transcribed spacer and $3^{\prime}$ to the $28 \mathrm{~S}$ region, which cross-hybridize to $5^{\prime}$ and $3^{\prime}$ flanking regions as well as to intron regions of rat a-fetoprotein, casein and serum albumin genes. These sequences are present in a minimum copy number of $1.2 \times 10^{5}$ per haploid genome.

The above findings are compatible with and support the model whereby transcription of structural genes produces stretches of RNA complementary to regions of the NTS of ribosomal genes. These stretches, which could be transcripts of the flanking gene sequences or of introns, upon processing of HnRNA, yield "signal" RNA, which moves to the nucleolar organizer, hybridizes with the respective NTS sequences and thus stimulates rDNA transcription. As HnRNA is complexed with a specific set of nuclear proteins (van Venrooij \& Janssen, 1978), it can be assumed that the "signal" RNA stretches would also be complexed to a particular class of these proteins. In analogy to recent findings on the role of snRNP complexes in splicing (for a review see Sekeris \& Guialis, 1981) the "signal" RNA associated proteins could be factors involved in polymerase I activation. Thus we can envisage that modulation of IDNA transcription could be attained both by a direct RNA/DNA interaction leading to structural alterations of the ribosomal genes, and by protein "activators" (Muramatsu, Shimada \& Higashinakawa, 1970; Yu \& Feigelson, 1972).

The model proposed conforms with the following observations: (a) all steroid hormones lead to a high degree of stimulation of HnRNA synthesis, although in most cases no bulk increase of cytoplasmic mRNA takes place; (b) in addition to HnRNA stimulation, the steroid hormones also stimulate rRNA synthesis, which follows the induction of HnRNA synthesis and (c) the high degree of glucocorticoid stimulation of repetitive sequences (Doenecke \& Sekeris, 1970).

The proposed hypothesis can be further experimentally tested, i.e. by labelling HnRNA in target tissues under basal conditions and after hormonal 
stimulation and looking for labelled, processed HnRNA stretches hybridizing with NTS.

\section{REFERENCES}

Arnheim, N., Sperack, P., Banerui, J., Lang, R. B., Miesfeld, R. \& Marcu, K. B. (1980). Cell 22, 179.

DAVIDSON, E. H. \& BRITTEN, R. (1979). Science 204, 1052.

DOENECKE, D. \& SEKERIS, C. E. (1970). FEBS Lett. 8,61.

GEORGIEV, G. P. (1969). J. theor. Biol. 25, 473.

Govindan, M. V., Spiess, E. \& Majors, J. (1982). Proc. nain. Acad. Sci. U.S.A. $79,5157$. JACOB, F. \& MONOD, J. (1961). J. mol. Biol. 3, 318.

Karlson, P., DoEnecke, D. \& SEKERIS, C. E. (1975). In Comprehensive Biochemistry, Vol. 25. (Florkin, E., ed.). New York: Academic Press.

Miesfeld, R., Krystal, M. \& ARnheim, N. (1981). Nucleic Acids Res. 9, 5931.

MroiczKa, D. L., Cassidy, B., Busch, H. \& Rothblum, L. I. (1984). J. mol. Biol. $174,141$.

Muramatsu, M., Shimada, N. \& Higashinakawa, T. (1970). J. mol. Biol. 53, 91.

Niessing, J., Schnieders, B., Kunz, W., Seifart, K. H. \& Sekeris, C. E. (1970). $Z$. Naturforsch. $25 b, 1119$.

NIESSING, J. \& SEKERIS, C. E. (1971). FEBS Lett. 247, 391.

Nomura, M., Gourse, R. \& Baugham, G. (1984). A. Rev. Biochem. 53, 75.

Scheidereit, C., Geisse, S., Westphal, H. M. \& BeAto, M. (1983). Nature 304, 749.

SCHMID, W. \& Sekeris, C. E. (1973a). Biochim. Biophys. Acta 312, 549.

SCHMID, W. \& SEKERIS, C. E. (1973b). FEBS Lett. 26, 109.

Sekeris, C. E. \& Guialis, A. (1981). In: The Cell Nucleus, Vol. VIII. (Busch, H., ed.) p. 247. New York: Academic Press.

YU, F.-L. \& FEIGELSON, P. (1972). Proc. natn. Acad. Sci. U.S.A. 69, 2833. 